\title{
An efficient battery lifetime aware power saving (EBLAPS) mechanism in IEEE 802.16e networks
}

\begin{abstract}
The IEEE 802.16e standard is an emergent broadband wireless access technology that added the mobility feature to the original standard. This feature made battery life of an operated mobile subscribe station (MSS) a bigger challenge because an MSS is powered by a rechargeable battery. The battery lifetime mechanism has to be created in order to prolong the battery-life of an MSS. The battery lifetime-aware power saving (BLAPS) scheme has been created to prolong the battery life of an MSS by adaptively adjusting the three-sleep parameters named idle threshold, initial sleep window, and final sleep window according to the residual energy and the traffic load. However, the scheme minimized the energy consumption of the MSS at the expense of the average response delay due to the effect of the remaining energy. It also used the standard sleep mode algorithm where the MSS frequently goes to listening mode when the traffic is low which leads to the high-energy consumption. In this paper, a new energy mechanism called efficient battery life-aware power saving scheme has been proposed to enhance the parameters of BLAPS. The three-sleep parameters in BLAPS mechanism are analytically enhanced according to the downlink stochastic traffic arrival pattern of an MSS. Moreover, an improved sleep mode control algorithm has been introduced to reduce the frequent transition to listening mode in case of low traffic. The simulation has been extensively used to evaluate the proposed scheme. The results have shown that the proposed scheme outperforms the BLAPS significantly in terms of both the average response delay and the average energy consumption.
\end{abstract}

Keyword: Efficient battery; IEEE 802.16e; Power saving scheme 